\title{
Real-time monitoring system for parking space management services
}

\begin{abstract}
Real-Time Monitoring System for Parking Space Management Services is the evolution of traditional parking system that it does not only provide live information to users in order to make it easy for them to look for vacant parking lot, it also give authority to operators to monitor and perform simulations to illustrate the real parking system. The purpose of this project is to apply the principles of queue theory into parking system modeling. The queue system model enables the predictions of arrival and service time in the system through analysis and calculations. Aside of it, Graphical User Interface (GUI) is also designed and integrated into the parking system in order to allow parking zone operators to monitor the status of the parking lots and view the statistics of arrival rate, service time and so on. This project focuses on the system modeling and software development and implementation. It is expected that this project will be able to assist parking zone operators in designing their system that will work efficiently and generate high income. It will also provide convenience to the operators in managing the parking zone remotely. Users will also experience a timesaving and stress-free parking zone.
\end{abstract}

Keyword: Queue model; Parking management; Graphical user interface 